\title{
Association of lipid profiles and the ratios with arterial stiffness in middle-aged and elderly Chinese
}

\author{
Weiwei Zhao ${ }^{1 \dagger}$, Wei Gong ${ }^{1+}$, Nan Wu' ${ }^{1}$, Yintao $\mathrm{Li}^{1}$, Kuanping Ye${ }^{1}$, Bin $\mathrm{Lu}^{1}$, Zhaoyun Zhang ${ }^{1}$, Shen $\mathrm{Qu}^{2}$, Yiming $\mathrm{Li}^{1}$, \\ Yehong Yang ${ }^{1 *}$ and Renming $\mathrm{Hu}^{1 *}$
}

\begin{abstract}
Background: Serum lipids and the ratios are known to be associated with the cardiovascular diseases (CVD). However, the associations of serum lipids and the ratios related to arterial stiffness are unclear. We sought to compare the strength of these serum lipids and the ratios with arterial stiffness assessing by brachial-ankle pulse wave velocity (baPW) in the middle-aged and elderly Chinese subjects.

Methods: A total number of 1133 Chinese aged from 50 to 90 years old were recruited from Shanghai downtown district. The serum lipids, baPWV and major cardiovascular risk factors of the participants were measured.

Results: Participants with high baPWV exhibited higher levels of non-HDL-C, TC/HDL-C, TG/HDL-C, LDL-C/HDL-c, and non-HDL-C/HDL-c, while HDL-c worked in the opposite direction (all $\mathrm{P}<0.05$ ). In addition, $T C, T G$, LDL-c, non-HDL-c, $\mathrm{TC} / \mathrm{HDL}-\mathrm{C}, \mathrm{TG} / \mathrm{HDL}-\mathrm{C}, \mathrm{LDL}-\mathrm{C} / \mathrm{HDL}-\mathrm{C}$, and non-HDL-C/HDL-c had a positive relationship with the baPWV value, while $\mathrm{HDL}-\mathrm{c}$ was on the contrary (all $\mathrm{P}<0.05)$. Finally, individuals with high non-HDL-C/HDL-c (OR $1.71,95 \% \mathrm{Cl} 1.06-2.55$, $\mathrm{P}=0.013)$ and low HDL-C (OR 0.57, 95\% Cl 0.35-0.96, $\mathrm{P}=0.024)$ were seem to be at high risk of arterial stiffness.

Conclusions: As a risk indicator, non-HDL-C/HDL-c, which could be readily obtained from routine serum lipids, was significantly associated with baPWV. Non-HDL-C/HDL-c was superior to traditional lipid variables for estimating arterial stiffness in the middle-aged and elderly Chinese population.
\end{abstract}

Keywords: Lipid profiles, Arterial stiffness, Brachial-ankle pulse wave velocity (baPWV)

\section{Introduction}

As cardiovascular diseases (CVD) have been the leading cause of death worldwide, the identification of cardiovascular risk factors at an early stage of life has become more and more popular. Some fixed factors (age, sex, and family history) and a number of modifiable factors (hyperlipidemia, smoking, hypertension, hyperglycemia, etc.) had been emerging for predicting CVD [1].

Dyslipidemia is characterized with elevated total cholesterol (TC), triglyceride (TG), and low-density lipoprotein cholesterol (LDL-c) as well as decreased high-density lipoprotein cholesterol (HDL-c) concentrations. The serum

\footnotetext{
* Correspondence: yangyehongfd@163.com; renminghu@fudan.edu.cn ${ }^{\dagger}$ Equal contributors

${ }^{1}$ Department of Endocrinology and Metabolism, Huashan Hospital, Fudan University, Shanghai, China

Full list of author information is available at the end of the article
}

lipid abnormalities are recognized as independent approximate risks for CVD. Many investigations had demonstrated the relationships between TC, TG, HDL-c, LDL-c and CVD [2-5]. Arterial stiffness measured by brachial-ankle pulse wave velocity (baPWV) is known to be a marker of subclinical atherosclerosis and serves as an independent risk predictor for CVD [6]. The identification of arterial stiffness in these high risk individuals before the development of clinical atherosclerosis would be of substantial clinical benefit.

We had identified serum HDL-c that had protective effect on arterial stiffness in middle aged and elderly Chinese [7]. Several other cross-sectional studies had also evaluated the strength of the relationships between arterial stiffness and single proatherogenic lipid or ratio, such as the LDL-c, non-HDL-c, and TG/HDL-c. However, the superiority of the lipid parameters was not 
confirmed and was questioned by different studies [8-10]. In one Chinese study, LDL-c was found independently associated with aortic stiffness [8]. Furthermore, a Netherlandish study supported the use of non-HDL-c as a superior predictor to LDL-c in identifying individuals for arterial stiffness [9]. An American study showed that TG/HDL-c was an independent determinant of arterial stiffness in adolescents and young adults [10]. On these premises, it prompted us to perform the present study to directly compare the association of these serum lipids to the ratios (including TC, TG, HDL-c, LDL-c, non-HDL-c, TC/HDL-c, TG/HDL-c, LDL-c/ HDL-c, and non-HDL-c/HDL-c) with arterial stiffness assessing by baPWV in the middle-aged and elderly Chinese population in an attempt to optimize the risk factors.

\section{Methods}

\section{Study subjects}

From September 2007 to August 2008, a large-scale local health-check program was launched by Huashan Hospital in Shanghai, China. All studied individuals came from the Jiangninglu community of the Jingan District and the Youyilu community of the Baoshan District in Shanghai. A multistage stratified cluster sampling method was carried out to select subjects from the two communities. We selected the units in neighborhood. Only participants aged over 50 years old were recruited from each household. All studied individuals were of southern Han Chinese. A total of 1492 eligible subjects participated in the study, 359 of them were excluded from the analysis for the usage of hypolipidemic agents or missing data. Written informed consents were obtained from all participants and the study was approved by the Institutional Review Board of Huashan Hospital.

Participants with the following conditions were excluded: nutritional derangements; anaemia; malignancy; thyroid dysfunction; pregnancy; breast-feeding; mental illness.

\section{Data collection}

The methodology used to obtain data of clinical and biochemical variables had been previously published [7]. In brief, a standardized questionnaire was performed by qualified internist to collect information such as age, gender, smoking, self-reported dyslipidemia, hypertension, and CVD.

All subjects were assessed after overnight fasting for at least 10 hours. The details of anthropometric measurements include height, weight, waist circumference, hip circumference, and blood pressure were carried by trained physicians using standard methods. Body mass index (BMI) was calculated as weight in kilograms divided by the square of height in meters.

\section{Laboratory measurements}

The measurements of fasting plasma glucose and hemoglobin A1c, fasting serum insulin, TC, TG, HDL-c, LDL-c, creatinine, uric acid, and white blood cell counts were performed in the centralized laboratory of Huashan Hospital $[7,11]$. Non-HDL-c was calculated by subtracting HDL-c from TC. Insulin resistance was estimated by homeostasis model assessment index-insulin resistance (HOMA-IR) [12]. Glomerular filtration rate (GFR; expressed in $\mathrm{ml} / \mathrm{min} / 1.73 \mathrm{~m}^{2}$ ) was calculated by CKDEPI equation [13].

Hypertension was defined as if blood pressure 140/ $90 \mathrm{mmHg}$ or greater or current use of antihypertensive drugs [14]. Metabolic syndrome (Mets) was defined based on the updated International Diabetes Federation (IDF) criteria for Asian-American [15].

\section{Measurements of baPWV}

BaPWV was measured by the VP-1000 Automatic Arteriosclerosis Measurement System (model BP-203 RPE-II, Colin Co, Ltd, Komaki, Japan). The details of the measurement, validity, and reproducibility have been reported previously $[16,17]$. The correlation coefficients of interobserver and intraobserver reproducibility were 0.98 and 0.87 , respectively. The device can record pulse waves in four cuffs, record the transmission time to arm and ankles, calculate the transmission distance from the right arm to each ankle according to body height, and automatically compute the baPWV values by transmission time and distance. Measurements were taken after the subject had a 5 min rest in a supine position at room temperature. Participants were advised to refrain from smoking, alcohol, caffeinated beverages and vigorous exercise for over 2 hours prior to the examination. The relationship between left and right baPWV was pretty high $(\mathrm{r}=0.955, \mathrm{P}<0.001)$ [7]. As a result, we used the mean of bilateral baPWV value during analysis.

\section{Statistical analysis}

All statistical analysis was performed using SPSS version 16.0 (SPSS, Chicago, IL, USA). Continuous variables were expressed as means \pm SD or median (interquartile range). Categorical variables were represented by frequency and percentage. The characteristics of the study population and lipid parameters according to baPWV tertiles were compared by ANOVA for continuous variables and chi-squared test for categorical variables. Correlation coefficients between lipid parameters and baPWV were calculated by partial correlation analysis. The odds ratios for high baPWV (defined as baPWV >75th percentile) $[7,18]$ were calculated by a multivariate logistic regression analysis in different lipid parameters. We stratified the lipids and the ratios into tertiles and estimated the odds 
ratios for developing arterial stiffness among different tertiles by using the first tertile as reference.

\section{Results}

General characteristics of the study participants

1133 participants were analyzed, among which 38.0\% (430/1133) were men, 62.0\% (703/1133) were women. Table 1 compared the demographic, anthropometric, and biochemical characteristics of the experimental population, which was divided into different gender subgroups. The levels of age, waist to hip ratio, diastolic blood pressure, TC/HDL-c, LDL-c/HDL-c, nonHDL-c/

Table 1 Characteristics of participants categorized by gender classification

\begin{tabular}{|c|c|c|c|c|}
\hline & Total & Male & Female & $\mathbf{P}$ \\
\hline Number & 1133 & 430 & 703 & \\
\hline Age (years) & $64.0 \pm 8.6$ & $65.4 \pm 8.7$ & $63.1 \pm 8.4$ & $<0.001$ \\
\hline Smoking (\%) & $246(21.7)$ & 136(31.6) & $110(15.6)$ & $<0.001$ \\
\hline $\begin{array}{l}\text { Self-reported } \\
\text { CVD (\%) }\end{array}$ & 239(21.1) & 83(19.3) & $156(22.2)$ & 0.279 \\
\hline Waist to hip ratio & $0.88 \pm 0.06$ & $0.91 \pm 0.06$ & $0.86 \pm 0.06$ & $<0.001$ \\
\hline $\begin{array}{l}\text { Body mass } \\
\text { index }\left(\mathrm{kg} / \mathrm{m}^{2}\right)\end{array}$ & $25.4 \pm 3.3$ & $25.2 \pm 3.2$ & $25.5 \pm 3.3$ & 0.278 \\
\hline $\mathrm{SBP}(\mathrm{mmHg})$ & $138.7 \pm 19.2$ & $139.0 \pm 18.8$ & $138.5 \pm 19.4$ & 0.630 \\
\hline $\mathrm{DBP}(\mathrm{mmHg})$ & $83.8 \pm 10.6$ & $85.0 \pm 10.1$ & $83.0 \pm 10.8$ & 0.002 \\
\hline Hypertension (\%) & 809(71.4) & $317(73.7)$ & 492(70.0) & 0.200 \\
\hline $\mathrm{TC}$ & $5.34 \pm 1.00$ & $5.08 \pm 0.91$ & $5.50 \pm 1.03$ & $<0.001$ \\
\hline TG & $1.61(1.09-2.30)$ & $1.55(1.06-2.20)$ & $1.65(1.10-2.38)$ & 0.154 \\
\hline $\mathrm{HDL}$ & $1.29 \pm 0.32$ & $1.21 \pm 0.31$ & $1.34 \pm 0.32$ & $<0.001$ \\
\hline LDL & $3.02 \pm 0.78$ & $2.91 \pm 0.73$ & $3.09 \pm 0.80$ & 0.001 \\
\hline nonHDL & $4.04 \pm 0.97$ & $3.87 \pm 0.91$ & $4.15 \pm 0.99$ & $<0.001$ \\
\hline TC/HDL & $4.32 \pm 1.11$ & $4.41 \pm 1.14$ & $4.27 \pm 1.09$ & 0.043 \\
\hline $\mathrm{TG} / \mathrm{HDL}$ & $1.29(0.80-2.05)$ & $1.36(0.86-2.11)$ & $1.27(0.77-1.98)$ & 0.613 \\
\hline LDL/HDL & $2.44 \pm 0.79$ & $2.53 \pm 0.84$ & $2.39 \pm 076$ & 0.005 \\
\hline nonHDL/HDL & $3.32 \pm 1.11$ & $3.41 \pm 1.14$ & $3.27 \pm 1.09$ & 0.044 \\
\hline FPG (mmol/l) & $6.9 \pm 2.3$ & $6.9 \pm 2.5$ & $6.8 \pm 2.1$ & 0.508 \\
\hline $\mathrm{HbA} 1 \mathrm{C}$ & $6.6 \pm 1.3$ & $6.8 \pm 1.5$ & $6.5 \pm 1.2$ & 0.021 \\
\hline HOMA-IR & $3.37(2.12-5.56)$ & $3.33(2.13-5.92)$ & $340(2.10-5.44)$ & 0.637 \\
\hline GFR (ml/min/1.732) & $92.2 \pm 13.7$ & $89.5 \pm 14.5$ & $93.9 \pm 13.0$ & $<0.001$ \\
\hline BaPW (cm/s) & $1745 \pm 364$ & $1782 \pm 364$ & $1723 \pm 362$ & 0.040 \\
\hline MetS (\%) & $759(67.0)$ & $247(57.4)$ & $512(72.8)$ & $<0.001$ \\
\hline $\begin{array}{l}\text { White blood } \\
\text { cell }\left(10^{9} /\right)\end{array}$ & $6.1 \pm 1.6$ & $6.4 \pm 1.7$ & $5.9 \pm 1.4$ & $<0.001$ \\
\hline $\begin{array}{l}\text { Serum creatinine } \\
(\mu \mathrm{mol} / \mathrm{l})\end{array}$ & $64.3 \pm 18.7$ & $76.4 \pm 21.7$ & $56.9 \pm 11.4$ & $<0.001$ \\
\hline Uric acid ( $\mu \mathrm{mol} / \mathrm{l})$ & $315(260-370)$ & $345(290-400)$ & $300(251-347)$ & 0.007 \\
\hline
\end{tabular}

CVD, cardiovascular disease; SBP, systolic blood pressure; DBP, diastolic blood pressure; FPG, fasting plasma glucose; HOMA-IR, homeostasis model assessment index-insulin resistance; GFR, glomerlar filtration rate; BaPWV, brachial-ankle pulse wave velocity; MetS, metabolic syndrome.

Data were reported in mean $\pm S D$, median (interquartile range), or number (percent).
HDL-c, HbA1C, white blood cell, serum creatinine, uric acid, and the percentage of smoking were significantly higher in men than in women. The levels of TC, HDL-c, LDL-c, nonHDL-c, GFR, baPWV, and the state of MetS were significantly lower in men than in women (all $\mathrm{P}<0.05$ ). The level of body mass index, systolic blood pressure, TG, TG/HDL-c, and the percentage of self-reported CVD, the state of hypertension were similar in both genders.

\section{Lipid profiles distribution according to baPWV tertiles}

The participants were divided into tertiles on the basis of the levels of baPWV. The lipid profiles and the ratios distribution of the participants differing by baPWV tertiles were performed in Table 2. In the overall and female population, participants with high baPWV exhibited higher levels of non-HDL-c, TC/HDL-c, TG/ HDL-c, LDL-c/HDL-c, and non-HDL-c/HDL-c, while HDL-c worked in the opposite direction $(\mathrm{P}<0.05)$. However, in men, only the levels of TC/HDL-c and nonHDL-c/HDL-c increased with the level of baPWV, and HDL-c decreased with the level of baPWV $(\mathrm{P}<0.05)$.

\section{The relationships between baPWV and lipid profiles}

As some lipid parameters were differently distributed in men and women, we investigated the relationships of serum lipids and lipid ratios with baPWV in men and woman separately. As shown in Table 3, baPWV was significantly associated with TC, TG, HDL-c, LDL-c, non-HDL-c, TC/HDL-c, TG/HDL-c, LDL-c/HDL-c, and non-HDL-c/HDL-c in both genders. The highest correlation coefficient reached 0.180 in non-HDL-c/HDL-c. However, the correlationship of TC and TG with baPWV disappeared in male, while the correlationship of TC and LDL-c with baPWV disappeared in female.

Remarkably, as presented in the multivariate logistic regression analysis, the ORs for probability of being arterial stiffness were increased along non-HDL-c/HDL-c (OR 1.71, 95\% CI 1.06-2.55, $\mathrm{P}=0.013$ ) tertiles after adjusting for age and sex, and decreased along HDL-c (OR 0.57, 95\% CI 0.35-0.96, P = 0.024) tertiles (Table 4). However, the ORs of TC, TG, LDL-c, non-HDL-c, TC/HDL-c, TG/HDL-c, and LDL-c/HDL-c did not reach statistical significance.

\section{Discussion}

The study was conducted in a population of middleaged and elderly Chinese, which was known to have a high prevalence of arterial stiffness. In previous study, we concluded that serum HDL-c had protective effect on arterial stiffness. As an extension of previous study, we investigated the clinical evaluation of the various lipid ratio profiles and had two major findings: 1) all the lipid profiles and lipid ratios, such as TC, TG, HDL-c, LDL-c, non-HDL-c, TC/HDL-c, LDL-c/HDL-c, 
Table 2 Serum lipids and their ratios distribution according to baPWV tertiles

\begin{tabular}{|c|c|c|c|c|c|}
\hline & & $\mathrm{T1}$ & $\mathrm{T} 2$ & T3 & $\mathbf{P}$ \\
\hline $\mathrm{TC}$ & Overall & $5.29 \pm 0.91$ & $5.40 \pm 0.98$ & $5.34 \pm 1.07)$ & 0.478 \\
\hline \multirow[t]{2}{*}{$(\mathrm{mmol} / \mathrm{L})$} & M & $5.06 \pm 0.79$ & $5.12 \pm 0.86$ & $5.05 \pm 0.95$ & 0.597 \\
\hline & $\mathrm{F}$ & $5.41 \pm 0.94$ & $5.55 \pm 1.01$ & $5.57 \pm 1.12$ & 0.307 \\
\hline TG & Overall & $1.51(1.01-2.17)$ & $1.69(1.03-2.37)$ & $1.58(1.11-2.47)$ & 0.045 \\
\hline \multirow[t]{2}{*}{$(\mathrm{mmol} / \mathrm{L})$} & M & $1.44(0.99-2.04)$ & $1.56(1.01-2.18)$ & $1.54(1.03-2.24)$ & 0.585 \\
\hline & $\mathrm{F}$ & $1.51(1.01-2.21)$ & $1.77(1.04-2.47)$ & $1.65(1.23-2.66)$ & 0.014 \\
\hline $\mathrm{HDL}-\mathrm{C}$ & Overall & $1.36 \pm 0.33$ & $1.29 \pm 0.30$ & $1.24 \pm 0.29$ & $<0.001$ \\
\hline \multirow[t]{2}{*}{$(\mathrm{mmol} / \mathrm{L})$} & M & $1.30 \pm 0.39$ & $1.26 \pm 0.31$ & $1.15 \pm 0.24$ & 0.019 \\
\hline & $\mathrm{F}$ & $1.39 \pm 0.30$ & $1.31 \pm 0.29$ & $1.30 \pm 0.31$ & 0.009 \\
\hline LDL-C & Overall & $2.98 \pm 0.72$ & $3.06 \pm 0.79$ & $2.98 \pm 0.76$ & 0.36 \\
\hline \multirow[t]{2}{*}{$(\mathrm{mmol} / \mathrm{L})$} & M & $2.84 \pm 0.66$ & $2.92 \pm 0.73$ & $2.88 \pm 0.73$ & 0.648 \\
\hline & $\mathrm{F}$ & $3.05 \pm 0.74$ & $3.14 \pm 0.82$ & $3.06 \pm 0.77$ & 0.442 \\
\hline nonHDL-c & Overall & $3.92 \pm 0.89$ & $4.11 \pm 0.97$ & $4.11 \pm 1.03$ & 0.043 \\
\hline \multirow[t]{2}{*}{$(\mathrm{mmol} / \mathrm{L})$} & M & $3.75 \pm 0.79$ & $3.86 \pm 0.92$ & $3.90 \pm 0.94$ & 0.512 \\
\hline & $\mathrm{F}$ & $4.00 \pm 0.93$ & $4.25 \pm 0.97$ & $4.27 \pm 1.07$ & 0.03 \\
\hline \multirow[t]{3}{*}{$\mathrm{TC} / \mathrm{HDL}-\mathrm{C}$} & Overall & $4.05 \pm 0.99$ & $4.35 \pm 1.07$ & $4.49 \pm 1.15$ & $<0.001$ \\
\hline & M & $4.14 \pm 1.05$ & $4.29 \pm 1.18$ & $4.56 \pm 1.14$ & 0.032 \\
\hline & $\mathrm{F}$ & $4.01 \pm 0.96$ & $4.39 \pm 1.02$ & $4.44 \pm 1.17$ & $<0.001$ \\
\hline \multirow[t]{3}{*}{ TG/HDL-C } & Overall & 1.09(0.68-1.89) & $1.35(0.77-2.03)$ & $1.37(0.86-2.19)$ & 0.003 \\
\hline & M & $1.21(0.74-2.08)$ & $1.33(0.77-1.95)$ & $1.40(0.84-2.24)$ & 0.187 \\
\hline & $\mathrm{F}$ & 1.07(0.63-1.83) & $1.38(0.78-2.08)$ & $1.33(0.86-2.17)$ & 0.005 \\
\hline \multirow[t]{3}{*}{ LDL-C/HDL-c } & Overall & $2.29 \pm 0.74$ & $2.47 \pm 0.78$ & $2.50 \pm 0.75$ & 0.004 \\
\hline & M & $2.35 \pm 0.78$ & $2.48 \pm 0.90$ & $2.58 \pm 0.78$ & 0.158 \\
\hline & $\mathrm{F}$ & $2.26 \pm 0.71$ & $2.47 \pm 0.71$ & $2.44 \pm 0.72$ & 0.012 \\
\hline \multirow[t]{3}{*}{ nonHDL-c/HDL-c } & Overall & $3.04 \pm 0.98$ & $3.40 \pm 1.07$ & $3.50 \pm 1.15$ & $<0.001$ \\
\hline & M & $3.13 \pm 1.05$ & $3.28 \pm 1.18$ & $3.56 \pm 1.14$ & 0.027 \\
\hline & $\mathrm{F}$ & $3.00 \pm 0.95$ & $3.39 \pm 1.02$ & $3.45 \pm 1.16$ & $<0.001$ \\
\hline
\end{tabular}

TC, total cholesterol; TG, triglyceride; HDL-c, high-density lipoprotein cholesterol.

LDL-c, low-density lipoprotein cholesterol; baPWV, brachial-ankle pulse wave velocity; T, tertile; $M$, male; F, female.

and non-HDL-c/HDL-c, are significantly correlated with arterial stiffness assessing by baPWV, and 2) Individuals with higher non-HDL-c/HDL-c levels have higher risk of arterial stiffness than other lipid parameters.

CVD represents a major cause of morbidity and mortality worldwide [1]. Arterial stiffness is an important determinant of cardiovascular risk $[19,20]$. Patients with hypercholesterolaemia have higher stiff blood vessels than matched controls, which have similar peripheral blood pressures [21]. Earlier studies had indicated that lipids and the related ratios were strong predictors of CVD. Some lipids had been the main target for clinical intervention of CVD [22-25]. Most previous studies focused on only one ratio or traditional lipids [8-10]. There is no study that evaluated the relationship between all the lipids and lipid ratios with arterial stiffness. In the present study, we evaluated the relationship between the lipids and the ratios with baPWV, and
Table 3 Correlation between baPWV and lipid parameters

\begin{tabular}{llll}
\hline Variable & Overall $^{\mathrm{b}}$ & Male $^{\mathrm{c}}$ & Female $^{\mathrm{c}}$ \\
\hline TC & $0.074^{\mathrm{a}}$ & 0.094 & 0.062 \\
$\mathrm{TG}$ & $0.124^{\mathrm{a}}$ & 0.102 & $0.132^{\mathrm{a}}$ \\
HDL-c & $-0.143^{\mathrm{a}}$ & $-0.140^{\mathrm{a}}$ & $-0.162^{\mathrm{a}}$ \\
LDL-c & $0.075^{\mathrm{a}}$ & $0.124^{\mathrm{a}}$ & 0.047 \\
nonHDL-c & $0.125^{\mathrm{a}}$ & $0.171^{\mathrm{a}}$ & $0.096^{\mathrm{a}}$ \\
TC/HDL-c & $0.177^{\mathrm{a}}$ & $0.231^{\mathrm{a}}$ & $0.130^{\mathrm{a}}$ \\
TG/HDL-c & $0.136^{\mathrm{a}}$ & $0.133^{\mathrm{a}}$ & $0.133^{\mathrm{a}}$ \\
LDL-C/HDL-c & $0.154^{\mathrm{a}}$ & $0.217^{\mathrm{a}}$ & $0.106^{\mathrm{a}}$ \\
Non-HDL-C/HDL-c & $0.180^{\mathrm{a}}$ & $0.240^{\mathrm{a}}$ & $0.135^{\mathrm{a}}$ \\
\hline
\end{tabular}

TC, total cholesterol; TG, triglyceride; HDL-c, high-density lipoprotein cholesterol.

LDL-c, low-density lipoprotein cholesterol; BaPWV, brachial-ankle pulse wave velocity.

${ }^{\mathrm{a}} \mathrm{P}<0.05$.

${ }^{\mathrm{b}}$ Correlation coefficients were calculated after adjusted for age and gender.

${ }^{\mathrm{c} C}$ Correlation coefficientswere calculated after adjusted for age. 
Table 4 Age and sex-adjusted odds ratios (95\% confidence intervals) for high baPWV by tertiles of lipid profiles

\begin{tabular}{|c|c|c|c|c|c|}
\hline \multirow[t]{3}{*}{ Tertiles } & \multicolumn{5}{|c|}{ Odds Ratio ( $95 \%$ confidence interval) } \\
\hline & \multicolumn{5}{|c|}{ Age and sex-adjusted } \\
\hline & TC & TG & $\mathrm{HDL}-\mathrm{C}$ & LDL-C & Non-HDL-C \\
\hline । & 1 & 1 & 1 & 1 & 1 \\
\hline$\|$ & $1.12(0.71-1.75)$ & 1.27(0.81-1.99) & $0.71(0.46-1.08)$ & $1.12(0.70-1.77)$ & $1.12(0.72-1.74)$ \\
\hline III & $1.27(0.85-1.92)$ & $1.38(0.91-2.11)$ & $0.57(0.35-0.96)$ & $1.40(0.90-2.16)$ & $1.27(0.84-2.04)$ \\
\hline \multirow[t]{2}{*}{$P$} & 0.501 & 0.185 & 0.024 & 0.104 & 0.394 \\
\hline & $\mathrm{TC} / \mathrm{HDL}-\mathrm{C}$ & $\mathrm{TG} / \mathrm{HDL}-\mathrm{C}$ & LDL-C/HDL-C & Non-HDL-c/HDL-c & \\
\hline । & 1 & 1 & 1 & 1 & \\
\hline$\|$ & $1.07(0.68-1.69)$ & $1.16(0.74-1.83)$ & 1.44(0.92-2.26) & $1.08(0.75-1.67)$ & \\
\hline III & $1.56(1.00-2.43)$ & $1.52(0.97-2.37)$ & $1.52(0.96-2.40)$ & $1.71(1.06-2.55)$ & \\
\hline$P$ & 0.050 & 0.069 & 0.075 & 0.013 & \\
\hline
\end{tabular}

TC, total cholesterol; TG, triglyceride; HDL-c, high-density lipoprotein cholesterol.

LDL-c, low-density lipoprotein cholesterol; BaPWV, brachial-ankle pulse wave velocity.

highlighted the rationale for using the lipid ratio of nonHDL-c/HDL-c as a risk factor for arterial stiffness risk in middle-aged and elderly Chinese.

In the study, HDL-c and all the lipid ratios were significantly correlated with the baPWV tertiles in the overall patients. Only HDL-c, TC/HDL-c, and non-HDL-c/ HDL-c worked in both the male and female population. All the conventional lipids and the ratios had a significant correlation with baPWV in the overall population. Only HDL-c and the lipid ratios worked in both the male and female population. Finally, in the multivariate logistic regression analysis after adjusting for age and gender, only non-HDL-c/HDL-c and HDL-c had significant estimating capacities. Non-HDL-c/LDL-c seemed to be superior to HDL-c in assessing the risk for arterial stiffness. Non-HDL-c/HDL-c, an easily obtained index, maybe offer convenience in screening large populations of arterial stiffness risk in routine clinical practice.

The pathophysiology of non-HDL-c/HDL-c with arterial stiffness is unclear, but data from literatures is helpful. Non-HDL-c is a measurement of the cholesterol in LDL and VLDL (intermediate-density lipoprotein and very-LDL) particles. In clinical practice, non-HDL-c had been recommended as a secondary therapeutic target in individuals with high TG concentration. It also had been reported to be a better predictor of CVD than LDL-c [26]. The non-HDL-c/HDL-c is a lineal combination of TG and HDL-c. In the last few years, the focus had been shifted towards non-HDL-c/HDL-c. A report from the Swedish National Diabetes Register demonstrated that non-HDL-c/HDL-c had a stronger relationship with coronary heart disease risk than LDL-c, HDL-c and nonHDL-c. A recent study found that non-HDL-c/HDL-c was a better indicator than apolipoprotein $\mathrm{B}$ (ApoB)/ apolipoprotein A1 (ApoA1) and ApoB/LDL-c in identifying MetS and insulin resistance $[27,28]$. However, few studies had evaluated the relationship between nonHDL-c/HDL-c ratio and arterial stiffness.

The study compared the association between the routine lipids and the related ratios with baPWV for the first time. However, the study has some limitations. First, the experimental population was modest in size, and entirely of Chinese origin, which may be limited in the generalizability of the results. Second, since the study was cross-sectional designed, no causal relationship between lipid profiles and the risk of arterial stiffness can be drawn. Thus, we hope the findings can stimulate more investigators.

In conclusion, the study provides the novel findings that all the lipids and the ratios are significantly correlated with baPWV. Non-HDL-c/HDL-c ratio performs more effectively in identifying individuals at increased arterial stiffness risk. As non-HDL-c/HDL-c is easy and cost-effective obtained, it seems reasonable to propose non-HDL-c/HDL-c as a surrogate indicator of arterial stiffness in clinical practice.

\footnotetext{
Abbreviations

baPW: Brachial-ankle pulse wave velocity; TC: Total cholesterol; TG: Triglyceride; LDL-c: Low-density lipoprotein cholesterol; HDL-c: High density lipoprotein-cholesterol; CVD: Cardiovascular diseases; BMI: Body mass index; HOMA-IR: Homeostasis model assessment index-insulin resistance; GFR: Glomerlar filtration rate; MetS: Metabolic syndrome; VLDL: Intermediate-density lipoprotein and very-LDL; ApoB: Apolipoprotein B; ApoA1: Apolipoprotein A1.
}

\section{Competing interests}

The authors declare that they have no competing interests.

\section{Authors' contributions}

YY and RH designed the study; WZ, NW, YL and KY participated in acquisition of data; $\mathrm{BL}, \mathrm{ZZ}, \mathrm{SQ}$ and $\mathrm{YL}$ researched and evaluated the literature; WZ and WG undertook the statistical analysis and wrote the first draft of the manuscript. All authors have approved the final manuscript for publication. 


\section{Acknowledgements}

This study was supported by the National Science Foundation of China $(81000329,81270902,81030014,3090069,81070238)$ and Key Project of Shanghai Science and Technology Committee (10JC140100230771024, 11DJ1400100)

\section{Author details}

${ }^{1}$ Department of Endocrinology and Metabolism, Huashan Hospital, Fudan University, Shanghai, China. ${ }^{2}$ Department of Endocrinology, Shanghai Tenth People's Hospital, School of medicine, Tongji University, Shanghai, China.

Received: 23 October 2013 Accepted: 11 February 2014

Published: 20 February 2014

\section{References}

1. Go AS, Mozaffarian D, Roger VL, Benjamin EJ, Berry JD, Borden WB, Bravata DM, Dai S, Ford ES, Fox CS, Franco S, Fullerton HJ, Gillespie C, Hailpern SM, Heit JA, Howard VJ, Huffman MD, Kissela BM, Kittner SJ, Lackland DT, Lichtman JH, Lisabeth LD, Magid D, Marcus GM, Marelli A, Matchar DB, McGuire DK, Mohler ER, Moy CS, Mussolino ME, Nichol G, Paynter NP, Schreiner PJ, Sorlie PD, Stein J, Turan TN, Virani SS, Wong ND, Woo D, Turner MB: American Heart Association Statistics Committee and Stroke Statistics Subcommittee: Heart disease and stroke statistics-2013 update: a report from the American Heart Association. Circulation 2013, 127:e6-e245.

2. Milionis HJ, Elisaf MS, Mikhailidis DP: Lipid abnormalities and cardiovascular risk in the elderly. Curr Med Res Opin 2008, 24:653-657.

3. Hosseini-Esfahani F, Mousavi Nasl Khameneh A, Mirmiran P, Ghanbarian A, Azizi F: Trends in risk factors for cardiovascular disease among Iranian adolescents: the Tehran Lipid and Glucose Study. J Epidemio/ 2011, 21:319-328.

4. De Freitas EV, Brandão AA, Pozzan R, Magalhães ME, Fonseca F, Pizzi O, Campana E, Brandão AP: Importance of high-density lipoprotein-cholesterol (HDL-C) levels to the incidence of cardiovascular disease (CVD) in the elderly. Arch Gerontol Geriatr 2011, 52:217-222.

5. Chan WB, Tong PC, Chow CC, So WY, Ng MC, Ma RC, Osaki R, Cockram CS, Chan JC: Triglyceride predicts cardiovascular mortality and its relationship with glycaemia and obesity in Chinese type 2 diabetic patients. Diabetes Metab Res Rev 2005, 21:183-188.

6. Hung CS, Lin JW, Hsu CN, Chen HM, Tsai RY, Chien YF, Hwang JJ: Using brachial-ankle pulse wave velocity to associate arterial stiffness with cardiovascular risks. Nutr Metab Cardiovasc Dis 2009, 19:241-246.

7. Zhao WW, Yang YH, Lu B, Feng XC, He M, Yang ZH, Wen J, Zhang ZY, Yang Z, Li Q, Ye Z, Gong W, Hu RM: Serum high-density lipoprotein cholesterol and progression to arterial stiffness in middle-aged and elderly Chinese. Nutr Metab Cardiovasc Dis 2013, 23:973-979.

8. Wang F, Ye P, Luo L, Xiao W, Qi L, Bian S, Wu H, Sheng L, Xiao T, Xu R: Association of serum lipids with arterial stiffness in a population-based study in Beijing. Eur J Clin Invest 2011, 41:929-936.

9. Holewijn S, Den Heijer M, Swinkels DW, Stalenhoef AF, De Graaf J: Apolipoprotein $B$, non-HDL cholesterol and LDL cholesterol for identifying individuals at increased cardiovascular risk. J Intern Med 2010, 268:567-577.

10. Di Bonito P, Moio N, Scilla C, Cavuto L, Sibilio G, Sanguigno E, Forziato C, Saitta F, lardino MR, Di Carluccio C, Capaldo B: Usefulness of the high triglyceride-to-HDL cholesterol ratio to identify cardiometabolic risk factors and preclinical signs of organ damage in outpatient children. Diabetes Care 2012, 35:158-162.

11. Li Q, Yang Z, Lu B, Wen J, Ye Z, Chen L, He M, Tao X, Zhang W, Huang Y, Zhang Z, Qu S, Hu R: Serum uric acid level and its association with metabolic syndrome and carotid atherosclerosis in patients with type 2 diabetes. Cardiovasc Diabetol 2011, 10:72.

12. Matthews DR, Hosker JP, Rudenski AS, Naylor BA, Treacher DF, Turner RC: Homeostasis model assessment: insulin resistance and beta-cell function from fasting plasma glucose and insulin concentrations in man. Diabetologia 1985, 28:412-419.

13. Levey AS, Stevens LA, Schmid $\mathrm{CH}$, Zhang $\mathrm{YL}$, Castro AF 3rd, Feldman HI, Kusek JW, Eggers P, Van Lente F, Greene T, Coresh J, CKD-EPI (Chronic Kidney Disease Epidemiology Collaboration): A new equation to estimate glomerular filtration rate. Ann Intern Med 2009, 150:604-612.

14. James PA, Oparil S, Carter BL, Cushman WC, Dennison-Himmelfarb C, Handler J, Lackland DT, Lefevre ML, Mackenzie TD, Ogedegbe O,
Smith SC Jr, Svetkey LP, Taler SJ, Townsend RR, Wright JT Jr, Narva AS, Ortiz E: 2014 Evidence-Based Guideline for the Management of High Blood Pressure in Adults: Report From the Panel Members Appointed to the Eighth Joint National Committee (JNC 8). JAMA 2014, 311:507-520.

15. Liu J, Grundy SM, Wang W, Smith SC Jr, Vega GL, Wu Z, Zeng Z, Wang W, Zhao D: Ethnic-specific criteria for the metabolic syndrome: evidence from China. Diabetes Care 2006, 29:1414-1416.

16. Yamashina A, Tomiyama H, Takeda K, Tsuda H, Arai T, Hirose K, Koji Y, Hori S, Yamamoto Y: Validity, reproducibility, and clinical significance of noninvasive brachial-ankle pulse wave velocity measurement. Hypertens Res 2002, 25:359-364.

17. Ohnishi H, Saitoh S, Takagi S, Ohata J, Isobe T, Kikuchi Y, Takeuchi H, Shimamoto K: Pulse wave velocity as an indicator of atherosclerosis in impaired fasting glucose: the Tanno and Sobetsu study. Diabetes Care 2003, 26:437-440.

18. Brinkley TE, Nicklas BJ, Kanaya AM, Satterfield S, Lakatta EG, Simonsick EM, Sutton-Tyrrell K, Kritchevsky SB: Plasma oxidized low-density lipoprotein levels and arterial stiffness in older adults: the health, aging, and body composition study. Hypertension 2009, 53:846-852.

19. Mattace-Raso FU, van der Cammen TJ, Hofman A, Van Popele NM, Bos ML, Schalekamp MA, Asmar R, Reneman RS, Hoeks AP, Breteler MM, Witteman $J C$ : Arterial stiffness and risk of coronary heart disease and stroke: the Rotterdam Study. Circulation 2006, 113:657-663.

20. Cohn JN: Arterial stiffness, vascular disease, and risk of cardiovascular events. Circulation 2006, 113:601-603.

21. Wilkinson I, Cockcroft JR: Cholesterol, lipids and arterial stiffness. Adv Cardiol 2007, 44:261-277.

22. Salazar MR, Carbajal HA, Espeche WG, Aizpurúa M, Leiva Sisnieguez CE, March CE, Balbín E, Stavile RN, Reaven GM: Identifying cardiovascular disease risk and outcome: use of the plasma triglyceride/high-density lipoprotein cholesterol concentration ratio versus metabolic syndrome criteria. J Intern Med 2013, 273:595-601.

23. Virani SS, Catellier DJ, Pompeii LA, Nambi V, Hoogeveen RC, Wasserman BA, Coresh J, Mosley TH, Otvos JD, Sharrett AR, Boerwinkle E, Ballantyne CM: Relation of cholesterol and lipoprotein parameters with carotid artery plaque characteristics: the Atherosclerosis Risk in Communities (ARIC) carotid MRI study. Atherosclerosis 2011, 219:596-602.

24. McQueen MJ, Hawken S, Wang X, Ounpuu S, Sniderman A, Probstfield J, Steyn K, Sanderson JE, Hasani M, Volkova E, Kazmi K, Yusuf S, INTERHEART study investigators: Lipids, lipoproteins, and apolipoproteins as risk markers of myocardial infarction in 52 countries (the INTERHEART study): a case-control study. Lancet 2008, 372:224-233.

25. Mora S, Glynn RJ, Boekholdt SM, Nordestgaard BG, Kastelein JJ, Ridker PM: On-treatment non-high-density lipoprotein cholesterol, apolipoprotein B, triglycerides, and lipid ratios in relation to residual vascular risk after treatment with potent statin therapy: JUPITER (justification for the use of statins in prevention: an intervention trial evaluating rosuvastatin). J Am Coll Cardiol 2012, 59:1521-1528

26. Denke MA: Weighing in before the fight: low-density lipoprotein cholesterol and non-high-density lipoprotein cholesterol versus apolipoprotein B as the best predictor for coronary heart disease and the best measure of therapy. Circulation 2005, 112:3368-3370.

27. Eliasson B, Cederholm J, Eeg-Olofsson K, Svensson AM, Zethelius B, Gudbjörnsdottir S, National Diabetes Register: Clinical usefulness of different lipid measures for prediction of coronary heart disease in type 2 diabetes: a report from the Swedish National Diabetes Register. Diabetes Care 2011, 34:2095-2100.

28. Kim SW, Jee JH, Kim HJ, Jin SM, Suh S, Bae JC, Kim SW, Chung JH, Min YK, Lee MS, Lee MK, Kim KW, Kim JH: Non-HDL-cholesterol/HDL-cholesterol is a better predictor of metabolic syndrome and insulin resistance than apolipoprotein B/apolipoprotein A1. Int J Cardiol 2013, 168:2678-2683.

\section{doi:10.1186/1476-511X-13-37}

Cite this article as: Zhao et al:: Association of lipid profiles and the ratios with arterial stiffness in middle-aged and elderly Chinese. Lipids in Health and Disease 2014 13:37. 